\title{
Design, Synthesis, Computer Modeling and Analgesic Activity of Some New Disubstituted Quinazolin-4(3H)-ones
}

\section{Rezk A Ayyad ${ }^{1,3}$, Helmy M Sakr ${ }^{1}$ and Kamal M El-Gamal ${ }^{2,3 *}$}

${ }^{1}$ Faculty of Pharmacy, Department of Pharmaceutical Chemistry, Al-Azhar University, Nasr City 11884, Cairo, Egypt

${ }^{2}$ Faculty of Pharmacy, Department of Organic Chemistry, Al-Azhar University, Nasr City 11884, Cairo, Egypt

${ }^{3}$ Faculty of Pharmacy, Department of Pharmaceutical Chemistry, Delta University, Gamsa, Dakahlia, Egypt

\begin{abstract}
A series of substituted quinazolin-4(3H)-ones $\left(\mathrm{VIII}_{1-12}\right.$ have been synthesized by treating 3-amino-2benzylamino-substituted- quinazolin-4(3H)-one $\mathrm{VII}_{1-4}$, with different aldehydes. The starting material 3-amino-2benzylamino substituted- quinazolin-4(3H)-one $\mathrm{VII}_{1-4}$ was synthesized by nucleophilic substitution of thiomethyl group of 3-amino-2-methylthio-substituted- quinazolin-4(3H)-one $\mathrm{VI}_{1-4}$ with benzylamine. The synthesized compounds $\mathrm{VIII}_{1-12}$ was investigated for analgesic activity. All the test compounds exhibited significant analgesic activity in comparison with paracetamol.
\end{abstract}

Keywords: 2,3-disubstituted quinazolin-4(3H)-one; Paracetamol; Modeling; Analgesic activity

Abbreviations: NSAIDs: Non-Steroidal Anti-Inflammatory Drugs; COX: Cyclooxygenase; DMSO: N,N-Dimethylformamide; PDB: Protein Data Bank; MOE: Molecular Operating Environment; MP: Melting Point.

\section{Introduction}

Quinazolines derivatives exhibited a vital role in many pharmacological activities [1-8] including anti-inflammatory, [9] antibacterial, [10] and anticonvulsant, [11] activities. Schiff's bases have generated a great deal of attention due to their interesting pharmaceutical activities include possess potent analgesic and antiinflammatory activities [12]. In the view of these facts and to develop earlier reporting [6] quinazoline-4(3H)-ones series that drawn great attention in the field of synthetic medicinal chemistry because it shown good analgesic and anti-inflammatory activities therefore, our aim was oriented to design derivatives of existing clinically used NSAIDs that has ability to inhibit the cyclooxygenase (COX) and as a result in safety when taking paracetamol [13] because it used in medication to treat pain and fever [14] through acting by inhibition of cyclooxygenase (COX), and recent findings suggest that it is highly selective for COX2 [15]. As part of our ongoing medicinal chemistry research program we found that quinazolines [6] especially quinazolin-4 $(3 \mathrm{H})$-ones with 2, 3-disubstitution that reported [16] to possess significant analgesic, anti-inflammatory activities. Based on these findings it is rationalized to synthesis and design new substituted quinazolin-4(3H)-ones and screen their anti-inflammatory and analgesic activities (Scheme 1).

\section{Experimental}

\section{General}

Chemistry: Melting points were measured in capillary tube on a Graffin melting point apparatus and are uncorrected. The IR spectra were recorded on Pye Unicam SP 1000 IR spectrophotometer using $\mathrm{KBr}$ discs $\left(\lambda_{\max }\right.$ in $\left.\mathrm{cm}^{-1}\right)$. ${ }^{1} \mathrm{HNMR}$ spectra were performed either on Gemini 300BB (300 MHz) or (500 MHz) and (300 MHz) for $\left.{ }^{13} \mathrm{C} \mathrm{NMR}\right)$, spectrometer, using TMS as internal standard and DMSO-d6 as solvent; the chemical shifts are reported in ppm $(\delta)$ and coupling constant $(J)$ values are given in Hertz $(\mathrm{Hz})$. Signal multiplicities are represented by $\mathrm{s}$ (singlet), $\mathrm{d}$ (doublet), $\mathrm{t}$ (triplet), q (quadruplet), and $\mathrm{m}$ (multiplet). All of the new compounds were analyzed for $\mathrm{C}, \mathrm{H}$ and $\mathrm{N}$ and agreed with the proposed structures within $\pm 0.4 \%$ of the theoretical values by the automated $\mathrm{CHN}$ analyzer. Mass spectra were recorded on Hewlett
Packard 5988 spectrometer at the RCMB. The purity of the compounds was checked by Thin Layer Chromatography (TLC) on Merck silica gel 60 F254 precoated sheets. All analyses were performed at the Microanalytical Unit of Cairo University, Cairo, Egypt. Starting Compounds 1-3 was prepared according to reported procedures [17-19].

Synthesis of substituted 3-Amino-2-mercapto Quinazolin4(3H)-one (V)

To a vigorously stirred solution of III1-4 derivatives (0.02 mol) in and hydrazine hydrate $95 \%$ (8.6 g, $0.2 \mathrm{~mol})$ that was added drop wise under cold condition. After the completion of addition, stirring was continued for $1.5 \mathrm{~h}$ at $50^{\circ} \mathrm{C}$ and the mixture was poured into icewater. The solid obtained was filtered, washed with water, then washed with absolute ethanol and crystallized from dimethylformamide then washed with ethanol to produce compound V.

3-amino-6-bromo-2-mercaptoquinazolin-4(3H)-one $\left(\mathrm{V}_{1}\right)$ : Yield: 68\%; MP: $228-230^{\circ} \mathrm{C}$; IR (KBr, $\left.v, \mathrm{~cm}^{-1}\right): 3300\left(\mathrm{NH}_{2}\right), 2560(\mathrm{SH}), 1700$ (C=O quinazoline ring), $1570(\mathrm{C}=\mathrm{N}) .{ }^{1} \mathrm{HNMR}(300 \mathrm{MHz},[\mathrm{D} 6]$ DMSO): $\delta=8.02\left(\mathrm{~s}, 1 \mathrm{H}, \mathrm{C}_{5}-\mathrm{H}\right), 7.76\left(\mathrm{~d}, 1 \mathrm{H}, \mathrm{J}=8.30 \mathrm{~Hz}, \mathrm{C}_{7}-\mathrm{H}\right), 7.43$ (s, $\left.1 \mathrm{H}, \mathrm{J}=16.52 \mathrm{~Hz}, \mathrm{C}_{8}-\mathrm{H}\right), 5.21\left(\mathrm{~s}, 2 \mathrm{H}, \mathrm{NH}_{2}, \mathrm{D}_{2} \mathrm{O}\right.$ exchangeable), 3.29 (s, $1 \mathrm{H}, \mathrm{SH}) .{ }^{13} \mathrm{C}$ NMR (300 MHz, [D6] DMSO): $\delta=121.6,123.2,124.7$, 132.5, 136.8, 146, 159.7, 160.7. MS (m/z): $274(\mathrm{M}+2,34.58 \%), 272(\mathrm{M}+$, 35, 11\%). Anal. Calcd. for $\mathrm{C}_{8} \mathrm{H}_{6} \mathrm{BrN}_{3}$ OS: C, 35.31; H, 2.22; N, 15.44 . Found: C, 35.17; H, 2.36; N, 15.62.

3-amino-6, 8-dibromo-2-mercaptoquinazolin-4(3H)-one $\left(\mathrm{V}_{2}\right)$ : Yield: $60 \%$; mp 237-239 ${ }^{\circ} \mathrm{C}$; IR $\left(\mathrm{KBr}, v, \mathrm{~cm}^{-1}\right): 3320\left(\mathrm{NH}_{2}\right), 2567(\mathrm{SH})$ $1690\left(\mathrm{C}=\mathrm{O}\right.$ quinazoline ring), $1573(\mathrm{C}=\mathrm{N}) .{ }^{1} \mathrm{HNMR}(300 \mathrm{MHz}$, [D6] DMSO): $\delta=7.93\left(\mathrm{~s}, 1 \mathrm{H}, \mathrm{C}_{5}-\mathrm{H}\right), 7.71\left(\mathrm{~s}, 1 \mathrm{H}, \mathrm{C}_{7}-\mathrm{H}\right), 5.41\left(\mathrm{~s}, 2 \mathrm{H}, \mathrm{NH}_{2}, \mathrm{D}_{2} \mathrm{O}\right.$ exchangeable), 3.21 (s, $1 \mathrm{H}, \mathrm{SH}) .{ }^{13} \mathrm{C}$ NMR (300 MHz, [D6] DMSO): $\delta=122,125.2,130.2,131.4,139.5,150.2,159.4,160.4 . \mathrm{MS}(\mathrm{m} / \mathrm{z}): 350$ $(\mathrm{M}+4,14.12 \%), 348(\mathrm{M}+2,28.44 \%), 272(\mathrm{M}+, 13.89 \%)$. Anal. Calcd.

*Corresponding author: Kamal M El-Gamal, Faculty of Pharmacy, Department of Organic Chemistry, Al-Azhar University, Nasr City 11884, Cairo, Egypt, E-mail: drkamalelgaml72@gmail.com

Received April 20, 2016; Accepted April 28, 2016; Published May 04, 2016

Citation: Ayyad RA, Sakr HM, El-Gamal KM (2016) Design, Synthesis, Computer Modeling and Analgesic Activity of Some New Disubstituted Quinazolin-4(3H)ones. Med chem (Los Angeles) 6: 299-305. doi:10.4172/2161-0444.1000360

Copyright: ( 2016 Ayyad RA, et al. This is an open-access article distributed under the terms of the Creative Commons Attribution License, which permits unrestricted use, distribution, and reproduction in any medium, provided the original author and source are credited. 


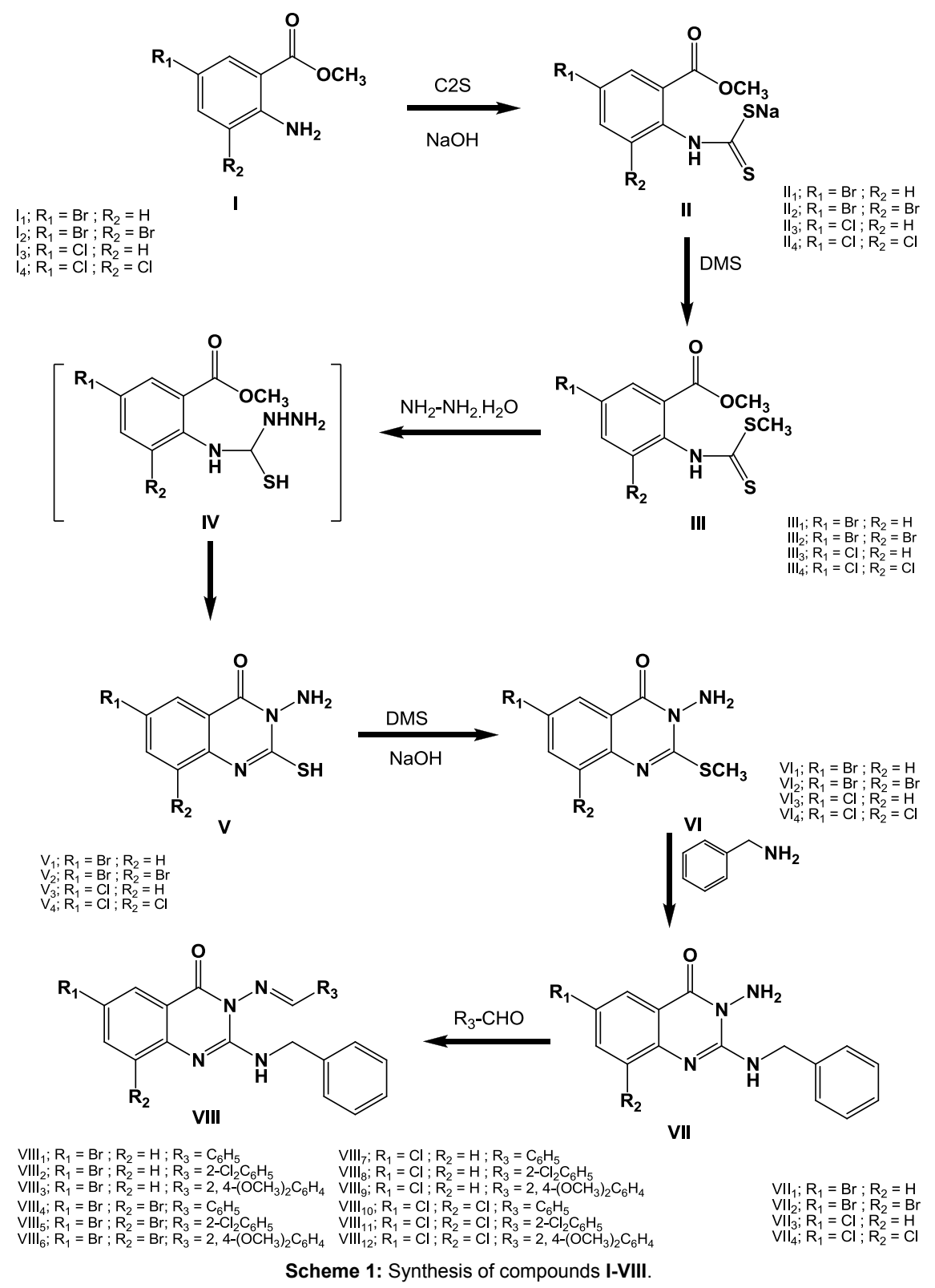

for $\mathrm{C}_{8} \mathrm{H}_{6} \mathrm{Br}_{2} \mathrm{~N}_{3} \mathrm{OS}$ : C, 27.37; $\mathrm{H}, 1.44 ; \mathrm{N}, 11.97$. Found: $\mathrm{C}, 27.48 ; \mathrm{H}, 1.66$; $\mathrm{N}, 11.72$.

3-amino-6-chloro-2-mercaptoquinazolin-4(3H)-one $\left(\mathrm{V}_{3}\right)$ : Yield: 75\%; MP: 204-206 ${ }^{\circ} \mathrm{C}$; IR (KBr, v, cm $\left.{ }^{-1}\right): 3280\left(\mathrm{NH}_{2}\right), 2570(\mathrm{SH}), 1700$ (C=O quinazoline ring), $1560(\mathrm{C}=\mathrm{N}) .{ }^{1} \mathrm{HNMR}(300 \mathrm{MHz},[\mathrm{D} 6] \mathrm{DMSO})$ : $\delta=7.93\left(\mathrm{~s}, 1 \mathrm{H}, \mathrm{C}_{5}-\mathrm{H}\right), 7.74\left(\mathrm{~d}, 1 \mathrm{H}, \mathrm{J}=7.50 \mathrm{~Hz}, \mathrm{C}_{7}-\mathrm{H}\right), 7.61(\mathrm{~d}, 1 \mathrm{H}, \mathrm{J}=$ $\left.8.60 \mathrm{~Hz}, \mathrm{C}_{8}-\mathrm{H}\right), 5.41$ (s, 2H, $\mathrm{NH}_{2}, \mathrm{D}_{2} \mathrm{O}$ exchangeable), 3.34 (s, 1H, SH). ${ }^{13} \mathrm{C}$ NMR (300 MHz, [D6] DMSO): $\delta=122.3,127.8,127.8,133,133.5$, 145, 159.4, 160.6. MS (m/z): 229 (M+2, 32\%), $227(\mathrm{M}+, 7.1 \%)$. Anal. Calcd. for $\mathrm{C}_{8} \mathrm{H}_{6} \mathrm{ClN}_{3} \mathrm{OS}$ : C, $42.20 ; \mathrm{H}, 2.66 ; \mathrm{N}, 18.46$. Found: $\mathrm{C}, 42.46$; $\mathrm{H}, 2.61 ; \mathrm{N}, 18.51$.

3-amino-6, 8-dichloro-2-mercaptoquinazolin-4(3H)-one $\left(\mathrm{V}_{4}\right)$ : Yield: $68 \%$; MP: $218-220^{\circ} \mathrm{C}$; IR $\left(\mathrm{KBr}, v, \mathrm{~cm}^{-1}\right): 3300\left(\mathrm{NH}_{2}\right), 2562$ (SH), $1700\left(\mathrm{C}=\mathrm{O}\right.$ quinazoline ring), $1572(\mathrm{C}=\mathrm{N}) .{ }^{1} \mathrm{HNMR}(300 \mathrm{MHz}$, [D6] DMSO): $\delta=7.93\left(\mathrm{~s}, 1 \mathrm{H}, \mathrm{C}_{5}-\mathrm{H}\right), 7.70\left(\mathrm{~s}, 1 \mathrm{H}, \mathrm{C}_{7}-\mathrm{H}\right), 5.69(\mathrm{~s}, 2 \mathrm{H}$, $\mathrm{NH}_{2}, \mathrm{D}_{2} \mathrm{O}$ exchangeable), 3.20 (s, 1H, SH). ${ }^{13} \mathrm{C}$ NMR (300 MHz, [D6]
DMSO): $\delta=123.7,125.9,129.4,134.4,135.2,159.2,159.4,160.7 . \mathrm{MS}$ $(\mathrm{m} / \mathrm{z}): 265(\mathrm{M}+4,1.94 \%), 263(\mathrm{M}+2,7.67 \%), 261(\mathrm{M}+, 10.2 \%)$. Anal. Calcd. for $\mathrm{C}_{8} \mathrm{H}_{5} \mathrm{Cl}_{2} \mathrm{~N}_{3} \mathrm{OS}$ : C, 36.66; $\mathrm{H}, 1.92 ; \mathrm{N}, 16.03$. Found: C, 36.71; $\mathrm{H}, 1.74 ; \mathrm{N}, 16.17$.

\section{Synthesis of substituted 3-Amino-2-methylthio Quinazolin-} 4(3H)-one (VI)

A solution of 3-amino-2-mercaptoquinazolin-4(3H)-one 1.93 $\mathrm{g}(0.01 \mathrm{~mol})$ in sodium hydroxide $10 \mathrm{ml}(20 \% \mathrm{w} / \mathrm{v})$ was obtained by warming on a water bath. It was clarified by filtration while in warm condition, cooled and treated with dimethyl sulphate $1.26 \mathrm{~g}(0.01 \mathrm{~mol})$ under constant stirring. The solution was stirred at room temperature for $12 \mathrm{~h}$. The solid obtained was filtered off, washed with cold water, dried and recrystallized from chloroform/ethanol.

3-amino-6-bromo-2-(methylthio) quinazolin-4(3H)-one ( $\left.\mathrm{VI}_{1}\right)$ : Yield: $80 \%$; MP: $172-174^{\circ} \mathrm{C}$; IR $\left(\mathrm{KBr}, v, \mathrm{~cm}^{-1}\right): 3320\left(\mathrm{NH}_{2}\right), 1700(\mathrm{C}=\mathrm{O}$ quinazoline ring), $1565(\mathrm{C}=\mathrm{N}) .{ }^{1} \mathrm{HNMR}(500 \mathrm{MHz},[\mathrm{D} 6] \mathrm{DMSO}): \delta$ 
$=8.20\left(\mathrm{~s}, 1 \mathrm{H}, \mathrm{C}_{5}-\mathrm{H}\right), 7.82\left(\mathrm{~d}, 1 \mathrm{H}, \mathrm{J}=8.0 \mathrm{~Hz}, \mathrm{C}_{7}-\mathrm{H}\right), 7.60(\mathrm{~d}, 1 \mathrm{H}, \mathrm{J}=$ $\left.8.0 \mathrm{~Hz}, \mathrm{C}_{8}-\mathrm{H}\right), 6.70\left(\mathrm{~s}, 2 \mathrm{H}, \mathrm{NH}_{2}, \mathrm{D}_{2} \mathrm{O}\right.$ exchangeable), 2.51 (s, $\left.3 \mathrm{H}, \mathrm{SH}_{3}\right)$. MS (m/z): $286(\mathrm{M}+2,21.12 \%), 284(\mathrm{M}+, 22.19 \%)$. Anal. Calcd. for $\mathrm{C}_{9} \mathrm{H}_{8} \mathrm{BrN}_{3} \mathrm{OS}: \mathrm{C}, 37.78 ; \mathrm{H}, 2.82 ; \mathrm{N}, 14.68$. Found: $\mathrm{C}, 37.92 ; \mathrm{H}, 2.90 ; \mathrm{N}$, 14.74 .

3-amino-6, 8-dibromo-2-(methylthio) quinazolin-4(3H)-one (VI $)$ : Yield: $72 \%$; MP: $186-188^{\circ} \mathrm{C}$; IR $\left(\mathrm{KBr}, \mathrm{v}, \mathrm{cm}^{-1}\right): 3325\left(\mathrm{NH}_{2}\right)$, $1680\left(\mathrm{C}=\mathrm{O}\right.$ quinazoline ring), $1570(\mathrm{C}=\mathrm{N}) .{ }^{1} \mathrm{HNMR}(500 \mathrm{MHz},[\mathrm{D} 6]$ DMSO): $\delta=8.20\left(\mathrm{~s}, 1 \mathrm{H}, \mathrm{C}_{5}-\mathrm{H}\right), 8.10\left(\mathrm{~s}, 1 \mathrm{H}, \mathrm{C}_{7}-\mathrm{H}\right), 6.62\left(\mathrm{~s}, 2 \mathrm{H}, \mathrm{NH}_{2}\right.$, $\mathrm{D}_{2} \mathrm{O}$ exchangeable), $2.55\left(\mathrm{~s}, 3 \mathrm{H}, \mathrm{SH}_{3}\right)$. MS (m/z): $366(\mathrm{M}+4,10.12 \%)$, $364(\mathrm{M}+2,20.44 \%), 362(\mathrm{M}+, 10.09 \%)$. Anal. Calcd. for $\mathrm{C}_{9} \mathrm{H}_{7} \mathrm{Br}_{2} \mathrm{~N}_{3} \mathrm{OS}$ : C, 29.61; H, 1.93; N, 11.51. Found: C, 29.72; H, 1.98; N, 11.42.

3-amino-6-chloro-2-(methylthio) quinazolin-4(3H)-one ( $\left.\mathrm{VI}_{3}\right)$ : Yield: 82\%; MP: $154-156^{\circ} \mathrm{C}$; IR $\left(\mathrm{KBr}, v, \mathrm{~cm}^{-1}\right): 3305\left(\mathrm{NH}_{2}\right), 1720(\mathrm{C}=\mathrm{O}$ quinazoline ring), $1575(\mathrm{C}=\mathrm{N}) .{ }^{1} \mathrm{HNMR}(500 \mathrm{MHz}$, [D6] DMSO): $\delta=$ $7.90\left(\mathrm{~s}, 1 \mathrm{H}, \mathrm{C}_{5}-\mathrm{H}\right), 7.69\left(\mathrm{~d}, 1 \mathrm{H}, \mathrm{J}=7.60 \mathrm{~Hz}, \mathrm{C}_{7}-\mathrm{H}\right), 7.40(\mathrm{~s}, 1 \mathrm{H}, \mathrm{J}=8.60$ $\left.\mathrm{Hz}, \mathrm{C}_{8}-\mathrm{H}\right), 6.72\left(\mathrm{~s}, 2 \mathrm{H}, \mathrm{NH}_{2}, \mathrm{D}_{2} \mathrm{O}\right.$ exchangeable), 2.50 (s, 3H, $\left.\mathrm{SH}_{3}\right) . \mathrm{MS}$ $(\mathrm{m} / \mathrm{z}): 243(\mathrm{M}+2,6.1 \%), 241(\mathrm{M}+, 17.9 \%)$. Anal. Calcd. for $\mathrm{C}_{9} \mathrm{H}_{8} \mathrm{ClN}_{3} \mathrm{OS}$ : C, 44.72; H, 3.34; N, 17.39. Found: C, 44.91; H, 3.52; N, 17.56.

3-amino-6, 8-dichloro-2-(methylthio) quinazolin-4(3H)-one (VI $)_{4}$ : Yield: $76 \%$; MP: $171-173^{\circ} \mathrm{C}$; IR $\left(\mathrm{KBr}, \mathrm{v}, \mathrm{cm}^{-1}\right): 3300\left(\mathrm{NH}_{2}\right)$, $1725\left(\mathrm{C}=\mathrm{O}\right.$ quinazoline ring), $1570(\mathrm{C}=\mathrm{N}) .{ }^{1} \mathrm{HNMR}(500 \mathrm{MHz},[\mathrm{D} 6]$ DMSO): $\delta=7.90\left(\mathrm{~s}, 1 \mathrm{H}, \mathrm{C}_{5}-\mathrm{H}\right), 7.80\left(\mathrm{~s}, 1 \mathrm{H}, \mathrm{C}_{7}-\mathrm{H}\right), 6.61\left(\mathrm{~s}, 2 \mathrm{H}, \mathrm{NH}_{2}\right.$, $\mathrm{D}_{2} \mathrm{O}$ exchangeable), $2.56\left(\mathrm{~s}, 3 \mathrm{H}, \mathrm{SH}_{3}\right)$. MS (m/z): $278(\mathrm{M}+4,1.11 \%)$, $276(\mathrm{M}+2,4.31 \%), 274(\mathrm{M}+, 5.20 \%)$. Anal. Calcd. for $\mathrm{C}_{9} \mathrm{H}_{7} \mathrm{Cl}_{2} \mathrm{~N}_{3} \mathrm{OS}: \mathrm{C}$, 39.15; H, 2.56; N, 15.22. Found: C, 39.41; H, 2.63; N, 15.39 .

\section{Synthesis of 3-Amino-2-substituted-benzylamino Quinazo- lin-4(3H)-one VII1-4}

A mixture of benzyl amine $5.35 \mathrm{~g}(0.05 \mathrm{~mol})$ and 3-amino-2methylthio substituted-quinazolin- $4(3 \mathrm{H})$-one $\mathrm{VI}_{1-4}(0.01 \mathrm{~mol})$ was heated under reflux at $80^{\circ} \mathrm{C}$ for $36 \mathrm{~h}$ then the reaction mixture was cooled and treated with petroleum ether. The solid product was obtained crystallized from ethanol $95 \%$ to afford the desired products $\mathrm{VII}_{1-4}$.

3-amino-2-(benzylamino)-6-bromoquinazolin-4(3H)-one (VII $)$ : Yield: $78 \%$; MP: $172-174^{\circ} \mathrm{C}$; IR $\left(\mathrm{KBr}, v, \mathrm{~cm}^{-1}\right): 3400\left(\mathrm{NH}_{2}\right)$, $1680\left(\mathrm{C}=\mathrm{O}\right.$ quinazoline ring), $1555(\mathrm{C}=\mathrm{N}) .{ }^{1} \mathrm{HNMR}(500 \mathrm{MHz},[\mathrm{D} 6]$ DMSO): $\delta=8.40\left(\mathrm{~s}, 1 \mathrm{H}, \mathrm{C}_{5}-\mathrm{H}\right), 8.05\left(\mathrm{~d}, 1 \mathrm{H}, \mathrm{J}=8.0 \mathrm{~Hz}, \mathrm{C}_{7}-\mathrm{H}\right), 7.75$ (d, $\left.1 \mathrm{H}, \mathrm{J}=7.50 \mathrm{~Hz}, \mathrm{C}_{8}-\mathrm{H}\right), 7.4-6.81$ (m, $5 \mathrm{H}$, aromatic protons), 5.80 (s, $2 \mathrm{H}, \mathrm{NH}_{2}, \mathrm{D}_{2} \mathrm{O}$ exchangeable), $4.87\left(\mathrm{~s}, 2 \mathrm{H}, \mathrm{CH}_{2}\right), 4.70(\mathrm{t}, 1 \mathrm{H}, \mathrm{NH}$, $\mathrm{D}_{2} \mathrm{O}$ exchangeable). Anal. Calcd. for $\mathrm{C}_{15} \mathrm{H}_{13} \mathrm{BrN}_{4} \mathrm{O}: \mathrm{C}, 52.19 ; \mathrm{H}, 3.80 ; \mathrm{N}$, 16.23. Found: C, 52.36; H, 3.56; N, 16.41 .

3-amino-2-(benzylamino)-6, 8-dibromoquinazolin-4(3H)-one (VII $)$ : Yield: 70\%; MP: 191-193 ${ }^{\circ} \mathrm{C}$; IR $\left(\mathrm{KBr}, v, \mathrm{~cm}^{-1}\right): 3395\left(\mathrm{NH}_{2}\right)$, 1690(C=O quinazoline ring), $1560(\mathrm{C}=\mathrm{N}) .{ }^{1} \mathrm{HNMR}(500 \mathrm{MHz},[\mathrm{D} 6]$ DMSO): $\delta=8.1-7.2\left(\mathrm{~m}, 7 \mathrm{H}\right.$, aromatic protons), $5.60\left(\mathrm{~s}, 2 \mathrm{H}, \mathrm{NH}_{2}, \mathrm{D}_{2} \mathrm{O}\right.$ exchangeable), $4.90\left(\mathrm{~s}, 2 \mathrm{H}, \mathrm{CH}_{2}\right), 4.40\left(\mathrm{t}, 1 \mathrm{H}, \mathrm{NH}, \mathrm{D}_{2} \mathrm{O}\right.$ exchangeable). Anal. Calcd. for $\mathrm{C}_{15} \mathrm{H}_{12} \mathrm{Br}_{2} \mathrm{~N}_{4} \mathrm{O}: \mathrm{C}, 42.48 ; \mathrm{H}, 2.85 ; \mathrm{N}, 13.21$. Found: $\mathrm{C}$, 42.66; H, 2.96; N, 13.39 .

3-amino-2-(benzylamino)-6-chloroquinazolin-4(3H)-one (VII $\left.{ }_{3}\right)$ : Yield: $75 \%$; MP: $160-162^{\circ} \mathrm{C}$; IR $\left(\mathrm{KBr}, v, \mathrm{~cm}^{-1}\right): 3390\left(\mathrm{NH}_{2}\right)$, $1688\left(\mathrm{C}=\mathrm{O}\right.$ quinazoline ring), $1540(\mathrm{C}=\mathrm{N}) .{ }^{1} \mathrm{HNMR}(500 \mathrm{MHz},[\mathrm{D} 6]$ DMSO): $\delta=8.20\left(\mathrm{~s}, 1 \mathrm{H}, \mathrm{C}_{5}-\mathrm{H}\right), 8.0\left(\mathrm{~d}, 1 \mathrm{H}, \mathrm{J}=7.0 \mathrm{~Hz}, \mathrm{C}_{7}-\mathrm{H}\right), 7.75$ $\left(\mathrm{d}, 1 \mathrm{H}, \mathrm{J}=7.0 \mathrm{~Hz}, \mathrm{C}_{8}-\mathrm{H}\right), 7.71-7.2(\mathrm{~m}, 5 \mathrm{H}$, aromatic protons), 5.40 (s, $2 \mathrm{H}, \mathrm{NH}_{2}, \mathrm{D}_{2} \mathrm{O}$ exchangeable), $4.60\left(\mathrm{~s}, 2 \mathrm{H}, \mathrm{CH}_{2}\right), 4.60\left(\mathrm{t}, 1 \mathrm{H}, \mathrm{NH}, \mathrm{D}_{2} \mathrm{O}\right.$ exchangeable). MS (m/z): $302(\mathrm{M}+2,3.2 \%), 300(\mathrm{M}+, 3.3 \%)$. Anal. Calcd. for $\mathrm{C}_{15} \mathrm{H}_{13} \mathrm{ClN}_{4} \mathrm{O}$ : C, 59.91; $\mathrm{H}, 4.36 ; \mathrm{N}, 18.63$. Found: C, 60.01; $\mathrm{H}, 4.46 ; \mathrm{N}, 18.91$. 3-amino-2-(benzylamino)-6, 8-dichloroquinazolin-4(3H)-one (VII ${ }_{4}$ ): Yield: $68 \%$; MP: $168-170^{\circ} \mathrm{C}$; IR $\left(\mathrm{KBr}, v, \mathrm{~cm}^{-1}\right): 3370\left(\mathrm{NH}_{2}\right)$, 1694(C=O quinazoline ring), $1558(\mathrm{C}=\mathrm{N}) .{ }^{1} \mathrm{HNMR}(500 \mathrm{MHz},[\mathrm{D} 6]$ DMSO): $\delta=8.23-7.50\left(\mathrm{~m}, 7 \mathrm{H}\right.$, aromatic protons), $5.80\left(\mathrm{~s}, 2 \mathrm{H}, \mathrm{NH}_{2}\right.$, $\mathrm{D}_{2} \mathrm{O}$ exchangeable), $4.80\left(\mathrm{t}, 1 \mathrm{H}, \mathrm{NH}, \mathrm{D}_{2} \mathrm{O}\right.$ exchangeable $), 4.60(\mathrm{~s}, 2 \mathrm{H}$, $\left.\mathrm{CH}_{2}\right)$. MS (m/z): $338(\mathrm{M}+4,1.8 \%), 336(\mathrm{M}+2,7.2 \%), 334(\mathrm{M}+, 9.01 \%)$. Anal. Calcd. for $\mathrm{C}_{15} \mathrm{H}_{12} \mathrm{Cl}_{2} \mathrm{~N}_{4} \mathrm{O}$ : C, 53.75; H, 3.61; N, 16.72. Found: C, 53.86; $\mathrm{H}, 3.76 ; \mathrm{N}, 16.86$.

\section{Synthesis of substituted- 2-Benzylamino-3-(substituted ben-} zylidene amino) quinazolin- $4(3 \mathrm{H})$-one $\mathrm{VIII}_{1-12}$

General procedure: A mixture of 3-amino-2-benzylamino substituted quinazolin-4(3H)-one $\mathrm{VII}_{1-4}(0.01 \mathrm{~mol})$ and different aromatic aldehydes derivative $(0.01 \mathrm{~mol})$ in acetic acid was refluxed for $30 \mathrm{hrs}$. After completion of reaction (TLC) the reaction mixture was poured into crushed ice and the solid obtained was crystallized from ethanol $95 \%$ to obtain pure compounds VIII ${ }_{1-12}$.

2-(benzylamino)-3-(benzylideneamino)-6-bromoquinazolin-4 (3H)-one (VIII $)$ : Yield: $82 \%$; MP: $165-167^{\circ} \mathrm{C}$; IR $\left(\mathrm{KBr}, \mathrm{v}, \mathrm{cm}^{-1}\right)$ : 3015 (CH-aromatic), $1688\left(\mathrm{C}=\mathrm{O}\right.$ quinazoline ring), $1550(\mathrm{C}=\mathrm{N}) .{ }^{1} \mathrm{HNMR}$ (300 MHz, [D6] DMSO): $\delta=8.65(\mathrm{~s}, 1 \mathrm{H}, \mathrm{CH}), 8.20-7.20(\mathrm{~m}, 13 \mathrm{H}$, aromatic protons), $4.90\left(\mathrm{~s}, 1 \mathrm{H}, \mathrm{NH}, \mathrm{D}_{2} \mathrm{O}\right.$ exchangeable), $4.10(\mathrm{~s}, 2 \mathrm{H}$, $\left.\mathrm{CH}_{2}\right) .{ }^{13} \mathrm{C}$ NMR (300 MHz, [D6] DMSO): $\delta=45,121.8,123.1,124.7$, $126.8,126.9,126.9,128.5,128.5,128.8,128.8,129.3,129.3,131.2,132.2$, $133.7,136.5,137,146,153.4,154.2,162.3$. MS (m/z): $434(\mathrm{M}+2,2.5 \%)$, $432(\mathrm{M}+, 2.51 \%)$. Anal. Calcd. for $\mathrm{C}_{22} \mathrm{H}_{17} \mathrm{BrN}_{4} \mathrm{O}: \mathrm{C}, 60.98 ; \mathrm{H}, 3.95 ; \mathrm{N}$, 12.93. Found: C, $60.84 ; \mathrm{H}, 3.84 ; \mathrm{N}, 12.97$.

2-(benzylamino)-6-bromo-3-(2-chlorobenzylide neamino) quinazolin-4(3H)-one (VIII $)$ ): Yield: $72 \%$; MP: $187-189^{\circ} \mathrm{C}$; IR $(\mathrm{KBr}, v$, $\left.\mathrm{cm}^{-1}\right)$ : 3010 (CH-aromatic), $1690(\mathrm{C}=\mathrm{O}$ quinazoline ring), $1550(\mathrm{C}=\mathrm{N})$. ${ }^{1} \mathrm{HNMR}(300 \mathrm{MHz},[\mathrm{D} 6]$ DMSO): $\delta=8.50(\mathrm{~s}, 1 \mathrm{H}, \mathrm{CH}), 8.30-7.20(\mathrm{~m}$, $12 \mathrm{H}$, aromatic protons), $4.70\left(\mathrm{~s}, 1 \mathrm{H}, \mathrm{NH}, \mathrm{D}_{2} \mathrm{O}\right.$ exchangeable), $3.90(\mathrm{~s}$, $\left.2 \mathrm{H}, \mathrm{CH}_{2}\right)$. MS (m/z): 469(M+2, 7.4\%), 467( $\left.\mathrm{M}+12.3 \%\right)$. Anal. Calcd. for $\mathrm{C}_{22} \mathrm{H}_{16} \mathrm{BrClN}_{4} \mathrm{O}: \mathrm{C}, 56.49 ; \mathrm{H}, 3.45 ; \mathrm{N}, 11.98$. Found: $\mathrm{C}, 56.71 ; \mathrm{H}$, $3.67 ; \mathrm{N}, 11.84$.

2-(benzylamino)-6-bromo-3-(2,4-dimethoxybenzylideneamino)quinazolin-4(3H)-one (VIII $)$ : Yield: $68 \%$; MP: $205-207^{\circ} \mathrm{C}$; IR ( $\mathrm{KBr}, v$, $\left.\mathrm{cm}^{-1}\right)$ : $3012(\mathrm{CH}$-aromatic), $1685(\mathrm{C}=\mathrm{O}$ quinazoline ring), $1551(\mathrm{C}=\mathrm{N})$. ${ }^{1} \mathrm{HNMR}$ (300 MHz, [D6] DMSO): $\delta=8.90(\mathrm{~s}, 1 \mathrm{H}, \mathrm{CH}), 8.00-7.20(\mathrm{~m}$, $11 \mathrm{H}$, aromatic protons), $4.68\left(\mathrm{~s}, 1 \mathrm{H}, \mathrm{NH}, \mathrm{D}_{2} \mathrm{O}\right.$ exchangeable), 3.92( $\mathrm{s}$, $\left.2 \mathrm{H}, \mathrm{CH}_{2}\right), 3.74\left(\mathrm{~s}, 6 \mathrm{H}, \mathrm{OCH}_{3}\right)$. MS (m/z): $494(\mathrm{M}+2,34.2 \%), 496(\mathrm{M}+$, 34.3\%). Anal. Calcd. for $\mathrm{C}_{24} \mathrm{H}_{21} \mathrm{BrN}_{4} \mathrm{O}_{3}$ : C, 58.43; $\mathrm{H}, 4.29 ; \mathrm{N}, 11.36$. Found: $\mathrm{C}, 58.57 ; \mathrm{H}, 4.37 ; \mathrm{N}, 11.54$.

2-(benzylamino)-3-(benzylideneamino)-6, 8-dibromoquinazolin4(3H)-one (VIII $)_{4}$ : Yield: 74\%; MP: $171-173^{\circ} \mathrm{C}$; IR $\left(\mathrm{KBr}, v, \mathrm{~cm}^{-1}\right): 3010$ ( $\mathrm{CH}$-aromatic), $1680\left(\mathrm{C}=\mathrm{O}\right.$ quinazoline ring), $1550(\mathrm{C}=\mathrm{N}) .{ }^{1} \mathrm{HNMR}$ (300 MHz, [D6] DMSO): $\delta=8.50(\mathrm{~s}, 1 \mathrm{H}, \mathrm{CH}), 8.25-7.20(\mathrm{~m}, 12 \mathrm{H}$, aromatic protons), $4.71\left(\mathrm{~s}, 1 \mathrm{H}, \mathrm{NH}, \mathrm{D}_{2} \mathrm{O}\right.$ exchangeable), $4.00(\mathrm{~s}, 2 \mathrm{H}$, $\left.\mathrm{CH}_{2}\right)$. MS (m/z): $512.2(\mathrm{M}+2,21.6 \%), 510(\mathrm{M}+, 22.3 \%)$. Anal. Calcd. for $\mathrm{C}_{22} \mathrm{H}_{16} \mathrm{Br}_{2} \mathrm{~N}_{4} \mathrm{O}$ : C, 51.59; H, 3.15; N, 10.94. Found: C, 51.68; H, 3.34; $\mathrm{N}, 11.12$.

2-(benzylamino)-6,8-dibromo-3-(2-chlorobenzylid eneamino) quinazolin-4(3H)-one (VIII $)_{5}$ ): Yield: $77 \%$; MP: $192-194^{\circ} \mathrm{C}$; IR (KBr, $\left.v, \mathrm{~cm}^{-1}\right): 3012(\mathrm{CH}$-aromatic), $1692(\mathrm{C}=\mathrm{O}$ quinazoline ring), 1555 $(\mathrm{C}=\mathrm{N}) .{ }^{1} \mathrm{HNMR}(300 \mathrm{MHz}$, [D6] DMSO): $\delta=9.10(\mathrm{~s}, 1 \mathrm{H}, \mathrm{CH}), 7.90-$ $6.80\left(\mathrm{~m}, 11 \mathrm{H}\right.$, aromatic protons), $4.65\left(\mathrm{~s}, 1 \mathrm{H}, \mathrm{NH}, \mathrm{D}_{2} \mathrm{O}\right.$ exchangeable), 3.90 (s, $2 \mathrm{H}, \mathrm{CH}_{2}$ ). ${ }^{13} \mathrm{C}$ NMR (300 MHz, [D6] DMSO): $\delta=45,113.2$, $122,125.4,126.7,126.7,126.9,127.2,128.5,128.5,130.5,131.4,132.4$, $133.5,134.5,137.5,137.9,139.5,143.5,150.3,153.5,162.3$. MS (m/z): 
$583(\mathrm{M}+4,11.2 \%), 548(\mathrm{M}+2,22.7 \%), 5546(\mathrm{M}+, 72.44 \%)$. Anal. Calcd. for $\mathrm{C}_{22} \mathrm{H}_{15} \mathrm{Br}_{2} \mathrm{ClN}_{4} \mathrm{O}: \mathrm{C}, 48.34 ; \mathrm{H}, 2.77 ; \mathrm{N}, 10.25$. Found: $\mathrm{C}, 48.41 ; \mathrm{H}$, $2.86 ; \mathrm{N}, 10.35$.

2-(benzylamino)-6, 8-dibromo-3-(2, 4-dimethoxybenzylid eneamino)-quinazolin-4(3H)-one (VIII $)$ : Yield: $86 \%$; MP: 231 $233^{\circ} \mathrm{C}$; IR ( $\left.\mathrm{KBr}, v, \mathrm{~cm}^{-1}\right): 3009$ ( $\mathrm{CH}$-aromatic), $1690(\mathrm{C}=\mathrm{O}$ quinazoline ring), $1550(\mathrm{C}=\mathrm{N}) .{ }^{1} \mathrm{HNMR}(300 \mathrm{MHz},[\mathrm{D} 6] \mathrm{DMSO}): \delta=8.85(\mathrm{~s}, 1 \mathrm{H}$, $\mathrm{CH}), 8.20-7.20\left(\mathrm{~m}, 10 \mathrm{H}\right.$, aromatic protons), $4.65\left(\mathrm{~s}, 1 \mathrm{H}, \mathrm{NH}, \mathrm{D}_{2} \mathrm{O}\right.$ exchangeable), $3.95\left(\mathrm{~s}, 2 \mathrm{H}, \mathrm{CH}_{2}\right), 3.66\left(\mathrm{~s}, 6 \mathrm{H}, \mathrm{OCH}_{3}\right) . \mathrm{MS}(\mathrm{m} / \mathrm{z}): 572$ $(\mathrm{M}+2,51.01 \%), 570(\mathrm{M}+, 52.5 \%)$. Anal. Calcd. for $\mathrm{C}_{24} \mathrm{H}_{20} \mathrm{Br}_{2} \mathrm{~N}_{4} \mathrm{O}_{3}: \mathrm{C}$, 50.37; H, 3.52; N, 9.79. Found: C, 50.52; H, 3.58; N, 9.83 .

2-(benzylamino)-3-(benzylideneamino)-6-chloroquin azolin4(3H)-one (VIII $)$ : Yield: $80 \%$; MP: $159-161^{\circ} \mathrm{C}$; IR $\left(\mathrm{KBr}, v, \mathrm{~cm}^{-1}\right): 3010$ ( $\mathrm{CH}$-aromatic), $1690\left(\mathrm{C}=\mathrm{O}\right.$ quinazoline ring), $1550(\mathrm{C}=\mathrm{N}) .{ }^{1} \mathrm{HNMR}$ (300 MHz, [D6] DMSO): $\delta=8.90(\mathrm{~s}, 1 \mathrm{H}, \mathrm{CH}), 8.30-7.16(\mathrm{~m}, 12 \mathrm{H}$, aromatic protons), $4.65\left(\mathrm{~s}, 1 \mathrm{H}, \mathrm{NH}, \mathrm{D}_{2} \mathrm{O}\right.$ exchangeable), $3.90(\mathrm{~s}, 2 \mathrm{H}$, $\left.\mathrm{CH}_{2}\right)$. MS (m/z): $425(\mathrm{M}+2,11.39 \%), 423(\mathrm{M}+, 34.3 \%)$. Anal. Calcd. for $\mathrm{C}_{22} \mathrm{H}_{16} \mathrm{Cl}_{2} \mathrm{~N}_{4} \mathrm{O}: \mathrm{C}, 62.42 ; \mathrm{H}, 3.81 ; \mathrm{N}, 13.24$. Found: $\mathrm{C}, 62.56 ; \mathrm{H}, 3.92 ; \mathrm{N}$, 13.36 .

2-(benzylamino)-6-chloro-3-(2-chlorobenzylidene amino) quinazolin-4(3H)-one (VIII) ${ }_{8}$ : Yield: $71 \%$; mp 172-174 ${ }^{\circ} \mathrm{C}$; IR $(\mathrm{KBr}, v$, $\left.\mathrm{cm}^{-1}\right)$ : $3018(\mathrm{CH}$-aromatic), $1690(\mathrm{C}=\mathrm{O}$ quinazoline ring), $1557(\mathrm{C}=\mathrm{N})$. ${ }^{1} \mathrm{HNMR}(300 \mathrm{MHz},[\mathrm{D} 6]$ DMSO): $\delta=8.97(\mathrm{~s}, 1 \mathrm{H}, \mathrm{CH}), 8.33-7.25(\mathrm{~m}$, $11 \mathrm{H}$, aromatic protons), $4.68\left(\mathrm{~s}, 1 \mathrm{H}, \mathrm{NH}, \mathrm{D}_{2} \mathrm{O}\right.$ exchangeable), $3.95(\mathrm{~s}$, $\left.2 \mathrm{H}, \mathrm{CH}_{2}\right)$. MS (m/z): $458(\mathrm{M}+2,19.3 \%), 456(\mathrm{M}+, 58.3 \%)$. Anal. Calcd. for $\mathrm{C}_{22} \mathrm{H}_{15} \mathrm{Cl}_{3} \mathrm{~N}_{4} \mathrm{O}$ : C, 57.73; H, 3.30; N, 12.24. Found: C, 57.87; H, 3.42; $\mathrm{N}, 12.27$.

2-(benzylamino)-6-chloro-3-(2,4-dimethoxybenzylide neamino)-quinazolin-4(3H)-one ( $\left.\mathrm{VIII}_{9}\right)$ : Yield: $80 \%$; MP: 212$214^{\circ} \mathrm{C}$; IR $\left(\mathrm{KBr}, v, \mathrm{~cm}^{-1}\right): 3010(\mathrm{CH}$-aromatic), $1688(\mathrm{C}=\mathrm{O}$ quinazoline ring), $1550(\mathrm{C}=\mathrm{N}) .{ }^{1} \mathrm{HNMR}(300 \mathrm{MHz},[\mathrm{D} 6] \mathrm{DMSO}): \delta=8.96(\mathrm{~s}, 1 \mathrm{H}$, $\mathrm{CH}), 8.20-7.00(\mathrm{~m}, 11 \mathrm{H}$, aromatic protons $), 4.65\left(\mathrm{~s}, 1 \mathrm{H}, \mathrm{NH}, \mathrm{D}_{2} \mathrm{O}\right.$ exchangeable), $3.87\left(\mathrm{~s}, 2 \mathrm{H}, \mathrm{CH}_{2}\right), 3.62\left(\mathrm{~s}, 6 \mathrm{H}, \mathrm{OCH}_{3}\right) . \mathrm{MS}(\mathrm{m} / \mathrm{z}): 450$ $(\mathrm{M}+2,3.19 \%), 448(\mathrm{M}+, 10.1 \%)$. Anal. Calcd. for $\mathrm{C}_{24} \mathrm{H}_{21} \mathrm{ClN}_{4} \mathrm{O}_{3}: \mathrm{C}$, $64.21 ; \mathrm{H}, 4.68 ; \mathrm{N}, 12.48$. Found: C, 64.35; H, 4.81; N, 12.60 .

2-(benzylamino)-3-(benzylideneamino)-6,8-dichloro quinazolin-4(3H)-one (VIII ${ }_{10}$ ): Yield: $87 \%$; MP: $201-203^{\circ} \mathrm{C}$; IR (KBr, $v$, $\left.\mathrm{cm}^{-1}\right)$ : $3010(\mathrm{CH}$-aromatic), $1680(\mathrm{C}=\mathrm{O}$ quinazoline ring), $1550(\mathrm{C}=\mathrm{N})$. ${ }^{1} \mathrm{HNMR}(300 \mathrm{MHz},[\mathrm{D} 6]$ DMSO): $\delta=8.65(\mathrm{~s}, 1 \mathrm{H}, \mathrm{CH}), 8.30-7.20(\mathrm{~m}$, $12 \mathrm{H}$, aromatic protons), $4.73\left(\mathrm{~s}, 1 \mathrm{H}, \mathrm{NH}, \mathrm{D}_{2} \mathrm{O}\right.$ exchangeable), $3.80(\mathrm{~s}$, $\left.2 \mathrm{H}, \mathrm{CH}_{2}\right) . \mathrm{MS}(\mathrm{m} / \mathrm{z}): 427(\mathrm{M}+4,5.9 \%), 425(\mathrm{M}+2,12.1 \%), 423(\mathrm{M}+$, 42.1\%). Anal. Calcd. for $\mathrm{C}_{22} \mathrm{H}_{16} \mathrm{Cl}_{2} \mathrm{~N}_{4} \mathrm{O}: \mathrm{C}, 62.42 ; \mathrm{H}, 3.81 ; \mathrm{N}, 13.24$. Found: C, 62.55; H, 3.61; N, 13.39.

2-(benzylamino)-6,8-dichloro-3-(2-chlorobenzylidene amino) quinazolin-4(3H)-one (VIII ${ }_{11}$ ): Yield: 79\%; MP: 207-209 ${ }^{\circ} \mathrm{C}$; IR (KBr, $v$, $\left.\mathrm{cm}^{-1}\right)$ : $3020(\mathrm{CH}$-aromatic), $1688(\mathrm{C}=\mathrm{O}$ quinazoline ring), $1557(\mathrm{C}=\mathrm{N})$. ${ }^{1} \mathrm{HNMR}$ (300 MHz, [D6] DMSO): $\delta=8.96(\mathrm{~s}, 1 \mathrm{H}, \mathrm{CH}), 8.35-7.25(\mathrm{~m}$, $11 \mathrm{H}$, aromatic protons), $4.73\left(\mathrm{~s}, 1 \mathrm{H}, \mathrm{NH}, \mathrm{D}_{2} \mathrm{O}\right.$ exchangeable), $4.01(\mathrm{~s}$, $\left.2 \mathrm{H}, \mathrm{CH}_{2}\right)$. MS (m/z): $461(\mathrm{M}+4,14.2 \%), 459(\mathrm{M}+2,17.2 \%), 457(\mathrm{M}+$, 14.7\%). Anal. Calcd. for $\mathrm{C}_{22} \mathrm{H}_{15} \mathrm{Cl}_{3} \mathrm{~N}_{4} \mathrm{O}: \mathrm{C}, 57.73 ; \mathrm{H}, 3.30 ; \mathrm{N}, 12.24$. Found: C, 57.82; H, 3.49; N, 12.39 .

2 - (benzylamino ) - 6,8-dichloro-3 - (2,4-dimethoxy benzylideneamino)-quinazolin-4(3H)-one ( VIII $\left._{12}\right)$ : Yield: $78 \%$; MP: $231-233^{\circ} \mathrm{C}$; IR $\left(\mathrm{KBr}, v, \mathrm{~cm}^{-1}\right): 3009$ (CH-aromatic), $1688(\mathrm{C}=\mathrm{O}$ quinazoline ring), $1542(\mathrm{C}=\mathrm{N}) .{ }^{1} \mathrm{HNMR}(300 \mathrm{MHz}$, [D6] DMSO): $\delta=$ $8.76(\mathrm{~s}, 1 \mathrm{H}, \mathrm{CH}), 8.13-7.02(\mathrm{~m}, 10 \mathrm{H}$, aromatic protons), $4.35(\mathrm{~s}, 1 \mathrm{H}, \mathrm{NH}$, $\mathrm{D}_{2} \mathrm{O}$ exchangeable), $3.93\left(\mathrm{~s}, 2 \mathrm{H}, \mathrm{CH}_{2}\right), 3.46\left(\mathrm{~s}, 6 \mathrm{H}, \mathrm{OCH}_{3}\right) \cdot{ }^{13} \mathrm{C} \mathrm{NMR}$ (300 MHz, [D6] DMSO): $\delta=49,56,56,101.6,106.8,109.2,123.7,126$, $126.8,127,127,128.7,128.7,129.4,133.1,134.3,135.2,137.8,143.4$,
$153.5,159.3,159.6,162.4,164$. MS (m/z): $487(\mathrm{M}+4,1.1 \%), 485(\mathrm{M}+2$ 5.7\%), $483(\mathrm{M}+, 7.7 \%)$. Anal. Calcd. for $\mathrm{C}_{24} \mathrm{H}_{20} \mathrm{Cl}_{2} \mathrm{~N}_{4} \mathrm{O}_{3}: \mathrm{C}, 59.64 ; \mathrm{H}$, 4.17; N, 11.59. Found: C, 59.80; H, 4.27; N, 11.70.

\section{Pharmacology}

All the newly synthesized compounds VIII $_{1-12}$ were preliminarily evaluated for their analgesic and Anti-inflammatory activities (using writhing test) using paracetamol as writhing protective stander. The analgesic activity of the newly synthesized compounds VIII compared to paracetamol (Sigma Chemical Co., St. Louis, MO, USA) as a reference was measured after and $30,60,90,120,150$, and 180 after p-benzoquinone (Aldrich) subcutaneous injection. All the tested compounds significantly showed highly percentage of protection against writhing compared with the control untreated group.

\section{Analgesic screening}

Adult albino mice of either sex weighing 20-25 gm which was obtained from animal house of Department of Pharmacology, Faculty of Pharmacy, Al-Azhar University, Cairo, Egypt. Mice's were divided into twelve groups; each group consists of six mice's per cage. The mice's were kept under constant temperature $30^{\circ} \mathrm{C}$ and 12 hours light/dark cycle. All animals were acclimatized in the animal facility for at least two weeks prior the experiments. The animals were kept fastened for 24 hours prior to the experiment, but they were allowed free access to water $[20,21]$. The animal experiments described below comply with the ethical principles and guidelines for the care and use of laboratory animals adopted by the National Egyptian Community. The equipment used was Dial micrometer model (120 - 1206 Baty, Sussex, England). The test compounds as well Paracetamol were suspended in water by the aid of few drops of Tween-80 (Sigma) to produce 2\% suspension. And p-benzoquinone (Aldrich) was dissolved in water for injection containing a few drops of Tween- 80 to produce $0.02 \%$ solution and was used as writhing inducer.

\section{Analgesic activity}

The analgesic action of some newly synthesized compound was determined using the writhing method on mice [22]. The mice were randomly arranged in groups each of 10 animal's one group was kept as control. The animals of another group were given paracetamol subcutaneously in a dose of $30 \mathrm{mg} / \mathrm{kg}$ body weight. Mice of the other groups were blindly injected subcutaneously with test compounds in a dose of $150 \mathrm{mg} / \mathrm{kg}$ body weight. After 30 minutes, each animal of each group was injected with $0.25 \mathrm{ml}$ of $0.02 \%$ aqueous solution of p-benzoquinone and was observed for writhing after 30, 60, 90, 120, 150 and 180 minutes. Animals protected from writhing were recorded in each group and the analgesic potency of the test compounds was determined as percentage of protection against writhing. The results are presented in Table 1.

\section{Molecular Modeling}

Docking studies were carried out to examine the analgesic effect of compounds VIII ${ }_{1-12}$ which subjected to docking using Molecular Operating Environment (MOE) program [23] on the 3D structure of the cyclooxygenase-2 enzyme (COX-2) in a trial to predict their analgesic action drugs and the aim of the flexible docking calculations is prediction of correct binding geometry for each binder.

\section{Preparation of the target protein}

The protein target needs to be prepared and modeled according to the format requirements of the docking algorithms used. Thus the required protein was downloaded from Protein Data Bank (PDB) (code 


\begin{tabular}{|c|c|c|c|c|c|c|c|c|c|c|c|c|c|c|c|}
\hline \multirow{3}{*}{ Comp. No. VIII } & \multirow{3}{*}{$\begin{array}{l}\text { Dose } \\
\mathrm{mg} / \mathrm{kg}\end{array}$} & \multirow{2}{*}{\multicolumn{6}{|c|}{$\%$ of mice showing abolished writhing }} & \multirow{3}{*}{ Comp. No. VIII } & \multirow{3}{*}{$\begin{array}{l}\text { Dose } \\
\mathrm{mg} / \mathrm{kg}\end{array}$} & \multicolumn{6}{|c|}{$\%$ of mice showing abolished writhing } \\
\hline & & \multicolumn{2}{|c|}{ Time (minutes) } & & & & & & & \multicolumn{6}{|c|}{ Time (minutes) } \\
\hline & & 30 & 60 & 90 & 120 & 150 & 180 & & & 30 & 60 & 90 & 120 & 150 & 180 \\
\hline $\begin{array}{l}\text { Paracetamol } \\
\text { (control) }\end{array}$ & 20 & 100 & 100 & 100 & 100 & 100 & 100 & $\begin{array}{l}\text { Paracetamol } \\
\text { (control) }\end{array}$ & 20 & 100 & 100 & 100 & 100 & 100 & 100 \\
\hline $\mathrm{VIII}_{1}$ & 150 & 100 & 100 & 100 & 100 & 100 & 100 & $\mathrm{VIIII}_{7}$ & 150 & 100 & 100 & 100 & 100 & 100 & 100 \\
\hline $\mathrm{VIII}_{2}$ & 150 & 100 & 100 & 100 & 100 & 100 & 100 & $\mathrm{VIIII}_{8}$ & 150 & 100 & 100 & 100 & 100 & 100 & 100 \\
\hline $\mathrm{VIIII}_{3}$ & 150 & 100 & 100 & 100 & 100 & 100 & 100 & $\mathrm{VIIII}_{9}$ & 150 & 100 & 100 & 100 & 100 & 100 & 100 \\
\hline $\mathrm{VIIII}_{4}^{3}$ & 150 & 100 & 100 & 100 & 100 & 100 & 100 & $\mathrm{VIIII}_{10}$ & 150 & 100 & 100 & 100 & 100 & 100 & 100 \\
\hline $\mathrm{VIII}_{5}$ & 150 & 100 & 100 & 100 & 100 & 100 & 100 & $\mathrm{VIIII}_{11}$ & 150 & 100 & 100 & 100 & 100 & 100 & 100 \\
\hline $\mathrm{VIIII}_{6}$ & 150 & 100 & 100 & 100 & 100 & 100 & 100 & $\mathrm{VIIII}_{12}$ & 150 & 100 & 100 & 100 & 100 & 100 & 100 \\
\hline
\end{tabular}

Table 1: The analgesic effect of paracetamol and tested compounds VIII $I_{1-12}$ in mice.

4COX) using discovery Studio 2.5 software. Water molecules were removed from downloaded protein. Crystallographic disorders and unfilled valence atoms were corrected using alternate conformations and valence monitor options. Protein was subjected to energy minimization by applying CHARMM force fields for charge, and MMFF94 force field for partial charge. Inflexibility of structure is obtained by creating fixed atom constraint. The binding site of the protein was defined and prepared for docking.

\section{Tested compounds preparation}

The designed compounds 2D structures were sketched using ChemBio Draw Ultra 14.0 and saved in MDL-SDfile format. SD file opened, 3D structures were protonated and energy minimized by applying CHARMM force fields for charge, and MMFF94 force field for partial charge, then prepared for docking by optimization of the parameters.

\section{Results and Discussion}

The present work, involves the synthesis of new derivatives of substituted- 2-Benzylamino-3-(substituted benzylidene amino) quinazolin-4(3H)-one $\mathrm{VIII}_{1-12}$ via starting with key intermediates through bromination and chlorination of methylanthranilate using reported method [17-19] to get compound $I_{1-4}$. Compound $I_{1-4}$ underwent reaction with carbon disulfide and sodium hydroxide to afford $\mathrm{II}_{1-4}$ that suspected to dimethylsulfate resulting the compound III $_{1-4}$ that when reacted with hydrazine hydrate it yielded compound $\mathrm{V}_{1-4}^{1-4}$. The structures of such new compounds were confirmed by both elemental and spectral analyses. The IR spectra of $\mathrm{V}_{1-4}$ in $\mathrm{KBr}$ showed carbonyl stretching around $1700 \mathrm{~cm}^{-1}$ in addition, to $\mathrm{NH}_{2}$ stretching around $3300 \mathrm{~cm}^{-1}$. The ${ }^{1} \mathrm{H}$ NMR spectra of $\mathrm{V}_{1-4}$ in DMSO-d6, showed singlet of one proton, at 3.20-3.29 ppm due to $\mathrm{SH}$ group and $\mathrm{NH}_{2}$ group appeared as abroad singlet at 5.21-5.69 ppm which is $\mathrm{D}_{2} \mathrm{O}$ exchangeable moreover, In the ${ }^{13} \mathrm{C}$-NMR spectra of these compounds showed $\mathrm{C}=\mathrm{O}$ peak at about $160 \mathrm{ppm}$ corresponded to carbonyl groups of quinazoline ring that confirm the cyclization of intermediate IV into V. Consequently compound VI was obtained from V upon treatment with dimethyl sulfate in sodium hydroxide and the structure of the resulting compounds clearly confirmed from ${ }^{1} \mathrm{HNMR}$ spectra that showed disappearance of SH signal and appearance of singlet signal around $2.5 \mathrm{ppm}$ belong $\mathrm{SCH}_{3}$. Frequently, replacement of alkylthio group at 2-position with benzyl amine in simple electrophilic substitution reaction it produce new quinazoline compounds $\mathrm{VII}_{1-4}$ where the elemental analysis and spectral data confirm the existence of this substitution reaction where the ${ }^{1} \mathrm{HNMR}$ of new compound contain two signals one at 4.40-4.80 ppm and another one at 5.40-5.80 ppm that exchangeable with $\mathrm{D}_{2} \mathrm{O}$ which belong to $\mathrm{NH}$ and $\mathrm{NH}_{2}$ respectively, also the structure of some the compounds were established from the spectral data of the resulting compounds. The title compounds substituted2-Benzylamino-3-(substituted benzylidene amino) quinazolin-4(3H)- one VIII ${ }_{1-12}$ were obtained by the condensation of amino group of 3-amino-2-substituted-benzylamino Quinazolin-4(3H)-one $\mathrm{VII}_{1-4}$ with a different aromatic aldehydes that afford new Schiff's bases. The assignment of the produced Schiff's bases were based on spectral and elemental analysis where ${ }^{1} \mathrm{H}-\mathrm{NMR}$ spectrum of all the compounds VIII $_{1-12}$ showed disappearance of signal due to $\mathrm{NH}_{2}$ group in addition, the ${ }^{1-\mathrm{H}}$-NMR spectrum of all the compounds VIII ${ }_{1-12}$ exhibit singlet of one proton around 8.50-8.97 ppm which belong the $(\mathrm{N}=\mathrm{CH})$ proton. The IR spectrum of titled compounds VIII ${ }_{1-12}$ showed the presence of peak carbonyl $(\mathrm{C}=\mathrm{O}), \mathrm{NH}$ and Aryl groups. Because titled compounds contain halogen atom(s) its mass spectrum showed molecular ion peaks corresponding to their molecular formula in addition, to its isotopic peak moreover, in some compounds containing two halogen the mass spectrum showed peaks of $\mathrm{M}+, \mathrm{M}+2$, and $\mathrm{M}+4$ that clearly observed and consequently, proven the resulting product. Finally, the structure of the newly synthesized product compounds VIII $_{1-12}$ was proven on the basis of their elemental and spectral data. From the previous mentioned discussion it was observed that our synthetic strategies adopted to obtain the newly synthesized quinazolin-4(3H)-one depending using whether simple synthetic procedure or simple reagent(s). The results of analgesic testing indicate that the test compounds exhibited excellent significant analgesic activity and docking study revealed that the synthesized compounds have potential analgesic activity and can be further optimized and developed as a lead compound. The rationalized steps depend on ligand based drug design particularly a molecular hybridization approach that involves the coupling of two or more groups with relevant biological properties.

\section{Docking Discussion}

The obtained results indicated that all studied ligand have similar position and orientation inside the putative binding site of the COX -2 enzyme. The selected compounds VIII ${ }_{12}, \mathrm{VIII}_{9}, \mathrm{VIII}_{3}$, and $\mathrm{VIII}_{11}$ showed good binding energies ranging from -37.18 to $-39.12 \mathrm{kcal} / \mathrm{mol}$ (Table 2).

The proposed binding mode of compound $\mathrm{VIII}_{2}$ (affinity value of $-43.80 \mathrm{kcal} / \mathrm{mol}$ and $2 \mathrm{H}$-bonds) is shown in Figure 1 . One carbonyl group formed a hydrogen bond with a distance of $2-12 \mathrm{~A}^{\circ}$ with Ser530. The chloride atom formed a further hydrogen bond with a distance of $2.30 \mathrm{~A}^{\circ}$ with the acidic proton of Arg120. Furthermore, the compound formed Pi-sigma interaction with Phe518 and with ser353. The proposed binding mode of compound VIII4 (affinity value of $-42.08 \mathrm{kcal} / \mathrm{mol}$ and $2 \mathrm{H}$-bonds) is shown in Figure 2. One carbonyl group formed a hydrogen bond with a distance of $1.80 \mathrm{~A}^{\circ}$ with ser530. One bromide atom of the ring formed a further hydrogen bond with a distance of $2.37 \mathrm{~A}^{\circ}$ with the acidic proton of Arg120. Furthermore, the compound formed Pi-sigma interaction with Phe518, Trp387 and with Ser353. The proposed binding mode of compound VIII8 (affinity value of -39.12 $\mathrm{kcal} / \mathrm{mol}$ and $2 \mathrm{H}$-bonds) is shown in Figure 3. One carbonyl group formed a hydrogen bond with a distance of $2.09 \mathrm{~A}^{\circ}$ with Ser530. The 
Citation: Ayyad RA, Sakr HM, El-Gamal KM (2016) Design, Synthesis, Computer Modeling and Analgesic Activity of Some New Disubstituted Quinazolin-4(3H)-ones. Med chem (Los Angeles) 6: 299-305. doi:10.4172/2161-0444.1000360

\begin{tabular}{|c|c|c|c|c|c|c|c|}
\hline Compound & $\boldsymbol{\Delta} \mathbf{G}$ & Compound & $\boldsymbol{\Delta G}$ & Compound & $\boldsymbol{\Delta} \mathbf{G}$ & Compound & $\boldsymbol{\Delta} \mathbf{G}$ \\
\hline $\mathrm{VIII}_{1}$ & -40.42 & $\mathrm{VIII}_{4}$ & -42.08 & $\mathrm{VIII}_{7}$ & -40.99 & $\mathrm{VIII}_{10}$ & -40.86 \\
\hline $\mathrm{VIII}_{2}$ & -43.80 & $\mathrm{VIII}_{5}$ & -43.80 & $\mathrm{VIII}_{8}$ & -39.84 & $\mathrm{VIII}_{11}$ & -39.12 \\
\hline $\mathrm{VIII}_{3}$ & -39.09 & $\mathrm{VIII}_{6}$ & -39.96 & $\mathrm{VIII}_{9}$ & -37.33 & $\mathrm{VIII}_{12}$ & -37.18 \\
\hline \multicolumn{6}{|c}{ Paracetamol $\Delta \mathrm{G}=49.12$} \\
\hline
\end{tabular}

Table 2: $\Delta \mathrm{G}$ for ligand VIII ${ }_{1-12}$.

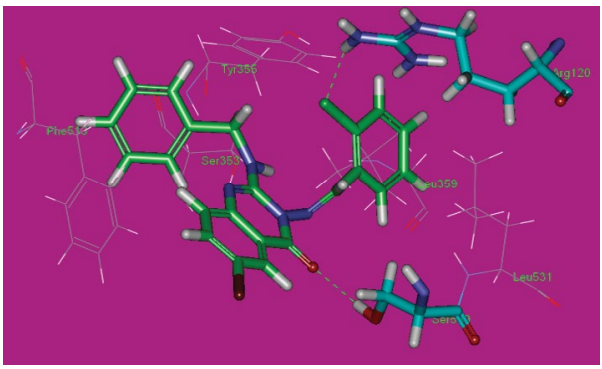

Figure 1: Binding mode of comp. $\mathrm{VIII}_{2}$.

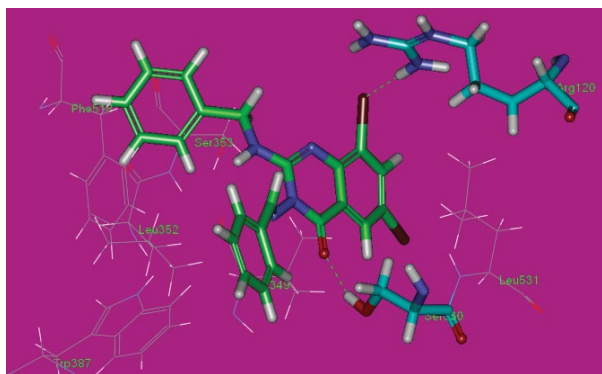

Figure 2: Binding mode of comp. VIII

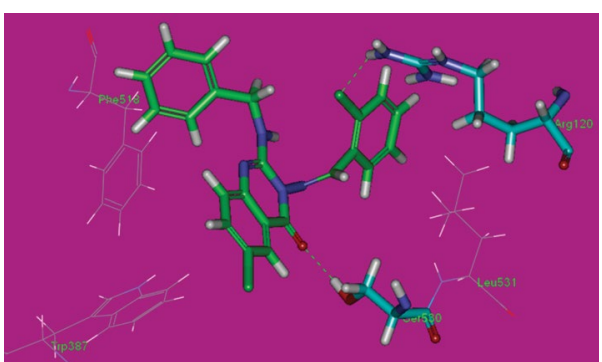

Figure 3: Binding mode of comp. VIII.

chloride atom of the side chain formed a further hydrogen bond with a distance of $2.32 \mathrm{~A}^{\circ}$ with the acidic proton of Arg120. Furthermore, the compound formed Pi-sigma interaction with Phe518 and with Trp387 (Figure 4).

\section{Conclusions}

We have synthesized newly derivatives of disubstituted quinazolin$4(3 \mathrm{H})$-ones that showed analgesic activity. From the data obtained in Table 1 it was found that all derivative VIII $_{1-12}$ have excellent significant analgesic activity. In addition to, the molecular docking for all compounds was performed on the active site of COX-2 enzyme in a trial to predict their mode of action as analgesic drugs, in which the compounds showed several interactions leading to the conclusion that they might exert their action through inhibition of COX-2 enzyme. The biological analgesic screening was performed in Pharmacology Department, Faculty of Pharmacy Al-Azher University, Cairo, Egypt.

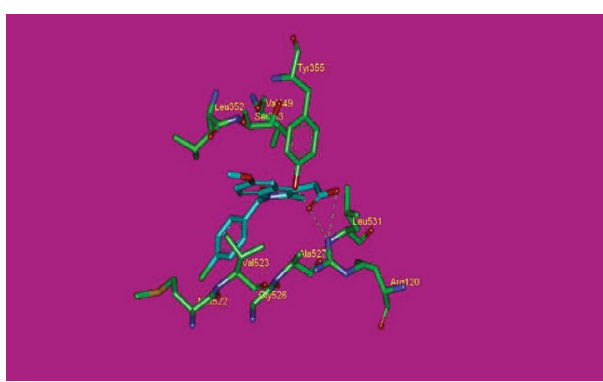

Figure 4: Binding mode of paracetamol.

\section{Acknowledgements}

The authors would like to express their sincere thanks to Dr. Ahmad Mansour, Pharmacology Department, Faculty of Pharmacy Al-Azher University, Cairo, Egypt for carrying out the analgesic activity for testing compounds. And I would like to thanks all members of Pharmacology Department, Faculty of Pharmacy Al-Azher University, Cairo, Egypt.

\section{References}

1. El-Azab AS, Abdel-Hamide SG, Sayed-Ahmed MM, Hassan GS El-Hadiyah TM, et al. (2013) Novel 4(3H)-quinazolinone analogs: synthesis and anticonvulsant activity. Med Chem Res 22: 2815-2827.

2. El-Azab AS, Eltahir KE (2012) Synthesis and anticonvulsant evaluation of some new 2,3,8-trisubstituted-4(3H)-quinazoline derivatives. Bioorg Med Chem Let 22: $327-333$.

3. Alagarsamy V, Shankar D, Solomon VR, Sheorey RV, Parthiban P (2009) Synthesis and pharmacological evaluation of 3-cyclohexyl-2-substituted hydrazino-3H-quinazolin-4-ones as analgesic and anti-inflammatory agents. Acta Pharm 59: 75-88.

4. Luo H, Hu D, Wu J, He M, Jin L, et al. (2012) Rapid synthesis and antiviral activity of (quinazolin-4-ylamino)methyl-phosphonates through microwave irradiation. Int J Mol Sci 13: 6730-6746.

5. Abdel Gawad NM, Georgey HH, Youssef RM, El-Sayed NA (2010) Synthesis and antitumor activity of some 2, 3-disubstituted quinazolin-4(3H)-ones and 4 6- disubstituted- 1, 2, 3, 4-tetrahydroquinazolin-2H-ones. Eur J Med Chem 45: 6058-6067.

6. Alagarsamy V, Raja Solomon V, Dhanabal K (2007) Synthesis and pharmacological evaluation of some 3-phenyl-2-substituted-3H-quinazolin-4 one as analgesic, anti-inflammatory agents. Bioorg Med Chem 15: 235-241.

7. Marvania B, Lee PC, Chaniyara R, Dong H, Suman S, et al. (2011) Design synthesis and antitumor evaluation of phenyl $\mathrm{N}$-mustard-quinazoline conjugates. Bioorg Med Chem 19: 1987-1998.

8. Heath JA, Mehrotra MM, Chi S, Yu JC, Hutchaleelaha A, et al. (2004) Identification of 4-piperazin-1-yl-quinazoline template based aryl and benzy thioureas as potent, selective, and orally bioavailable inhibitors of plateletderived growth factor (PDGF) receptor. Bioorg Med Chem Lett 14: 4867-4872.

9. Chandrika PM, Yakaiah T, Rao AR, Narsaiah B, Reddy NC, et al. (2008) Synthesis of novel 4,6-disubstituted quinazoline derivatives, their antiinflammatory and anti-cancer activity (cytotoxic) against U937 leukemia cell lines. Eur J Med Chem 43: 846-852.

10. Jatav V, Kashaw S, Mishra P (2008) Synthesis, antibacterial and antifungal activity of some novel 3-[5-(4-substituted phenyl),3,4-thiadiazole-2-yl]-2-styryl quinazoline-4(3H)-ones. Med Chem Res 17: 169-181.

11. Jatav V, Mishra P, Kashaw S, Stables JP (2008) CNS depressant and anticonvulsant activities of some novel 3-[5-substituted 1,3,4-thiadiazole-2-yl]2-styryl quinazoline-4(3H)-ones. Eur J Med Chem 43: 1945-1954.

12. Alafeefy AM, Kadi AA, Al-Deeb OA, El-Tahir KE, Al-Jaber NA (2010) Synthesis analgesic and anti-inflammatory evaluation of some novel quinazoline derivatives. Eur J Med Chem 45: 4947-4952.

13. Machado GC, Maher CG, Ferreira PH, Pinheiro MB, Lin CW, et al. (2015) Efficacy and safety of paracetamol for spinal pain and osteoarthritis: systematic review and meta-analysis of randomised placebo controlled trials. BMJ 350: h1225.

14. Graham GG, Davies MJ, Day RO, Mohamudally A, Scott KF (2013) The 
Citation: Ayyad RA, Sakr HM, El-Gamal KM (2016) Design, Synthesis, Computer Modeling and Analgesic Activity of Some New Disubstituted Quinazolin-4(3H)-ones. Med chem (Los Angeles) 6: 299-305. doi:10.4172/2161-0444.1000360

modern pharmacology of paracetamol: therapeutic actions, mechanism of action, metabolism, toxicity and recent pharmacological findings. Inflammopharmacology 21: 201-232.

15. Hinz B, Cheremina O, Brune K (2008) Acetaminophen (paracetamol) is a selective cyclooxygenase-2 inhibitor in man. FASEB J 22: 383-390.

16. Rajveer $\mathrm{CH}$, Swarnalatha $\mathrm{CH}$, Rathinaraj BS, Sudharshini S (2010) Synthesis of 6-bromo-oxo quinazoline derivatives and their pharmacological activities. International Journal of Chemical Research 1: 21-24.

17. Hemalatha K, Girija K (2011) Synthesis of some novel 2, 3-disubstituted quinazolinone derivatives as analgesic and anti-inflammatory agents. Int Pharm Pharm Sci 3: 103-106.

18. Kumar A, Sharma S, Archana, Bajaj K, Sharma S, et al. (2003) Some new 2,3,6-trisubstituted quinazolinones as potent anti-inflammatory, analgesic and COX-II inhibitors. Bioorg Med Chem 11: 5293-5299.
19. Balakumar C, Lamba P, Kishore DP, Narayana BL, Rao KV, et al. (2010) Synthesis, anti-inflammatory evaluation and docking studies of some new fluorinated fused quinazolines. Eur J Med Chem 45: 4904-4913.

20. Viegas-Junior C, Danuello A, da Silva Bolzani V, Barreiro EJ, Fraga CA (2007) Molecular hybridization: a useful tool in the design of new drug prototypes. Curr Med Chem 14: 1829-1852.

21. Koster R, Anderson M, Beer D (1959) Acetic acid-induced analgesic screening Federation Proceedings 18: 412.

22. Eckhardt ET, Cheplovitz F, Lipo M, Govier WM (1958) Etiology of chemically induced writhing in mouse and rat. Proc Soc Exp Biol Med 98: 186-188.

23. Chavan HV, Bandgar BP, Adsul LK, Dhakane VD, Bhale PS, et al. (2013) Design, synthesis, characterization and anti-inflammatory evaluation of novel pyrazole amalgamated flavones. Bioorg Med Chem Lett 23: 1315-1321. 